\title{
To bee or not to bee ... a nurse
}

In honeybee colonies, genotype does not determine phenotype; whether a bee becomes a queen or a worker depends on its diet during development, and worker bees can be either nurses or foragers, with markedly different behaviours. Herb et al. now show that the transition between nursing and foraging is associated with reversible alterations in gene methylation in the honeybee brain.

When worker bees emerge from pupae, they behave as nurses, caring

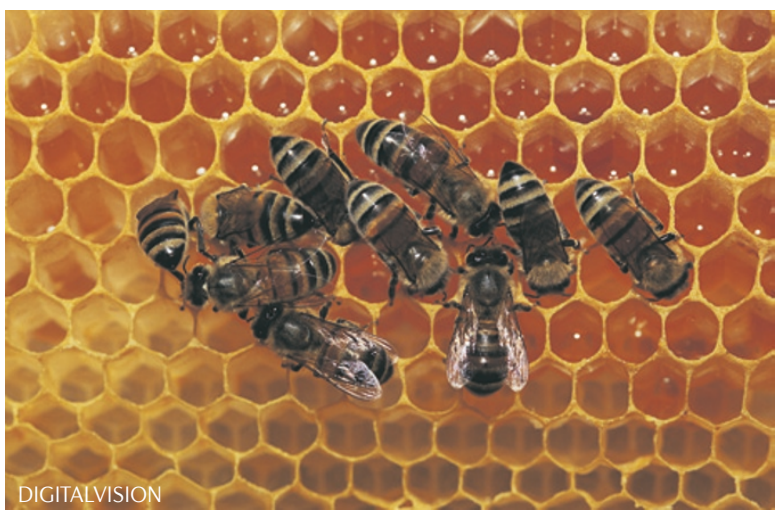

for larvae within the hive. After 2-3 weeks, most become foragers, venturing outside the hive to collect food and water, and this transition is associated with changes in gene expression in the brain. However, if forager bees are placed in a hive that contains only a queen and larvae, a subset of the foragers revert to nursing. Herb et al. took advantage of this behavioural reversal to investigate epigenetic differences between foragers and nurses.

The authors used two techniques to compare the methylomes of queen, nursing and foraging bees. Newly emerged queen and worker (nurse) bees showed no significant differences in methylation in the brain but age-matched nurse and forager bees showed marked differences; the authors found 155 'differentially methylated regions' (DMRs) that distinguished the two.

When forager bees were induced to revert to nursing and compared with age-matched foragers that had not reverted, the authors found 107 DMRs, around half of which matched those identified in the initial nurse-to-forager comparison (and therefore represent reversal of the initial nurse-to-forager methylation changes). These regions contained many genes involved in development and ATP-binding but also included genes involved in learning and axon migration.

The authors think that these results represent the first example, in any species, of a reversible DNA methylation pattern that corresponds to a reversible behavioural phenotype. The findings help to demonstrate the importance of epigenetic changes not just in development but also in behavioural switches during adulthood.

Rachel Jones

ORIGINAL RESEARCH PAPER Herb, B. R. et al.

Reversible switching between epigenetic states in honeybee behavioural subcastes. Nature Neurosci.

16 Sep 2012 (doi:10.1038/nn.3218) 